\title{
A (in)visibilidade gênero no currículo e na prática de duas especialidades médicas
}

\author{
Gender (in)visibility in the curriculum and in the practice of two medical specialities
}

\author{
Marcia Thereza Couto' (D) marthet@usp.br \\ Rosana Machin ${ }^{1}$ (D) rmachin@usp.br \\ Maria Renata Mencacci Costa ${ }^{1}$ (D) mrmencacci@hotmail.com \\ Ana Luisa Smith Rocha' (D) ana_rocha96@hotmail.com \\ Antônio Augusto Modesto² (1) filomedlit@yahoo.com.br \\ Ana Claudia Camargo Germani¹ (1) accggermani@usp.br
}

\begin{abstract}
RESUMO
Introdução: A medicina como área de conhecimento e prática tem invisibilizado a importância de gênero como categoria teórica na formação médica, bem como os impactos das diferenças e desigualdades de gênero expressas no contexto da prática clínica. Gênero é reconhecido como um aspecto crucial na educação médica, principalmente no sentido de promover a qualidade da assistência à saúde, considerando as diferenças de gênero nos sintomas, os fatores de risco da doença e o plano de assistência estabelecido no contexto da relação terapêutica.

Objetivo: Com base nesse pressuposto, foi realizada pesquisa sobre a percepção da formação recebida sobre gênero no contexto da graduação e especialização médica de residentes em ginecologia e obstetrícia e medicina de família e comunidade de duas escolas públicas do município de São Paulo.

Método: A pesquisa de abordagem qualitativa utilizou a técnica de entrevista em profundidade. Em 2016, 13 residentes de ambas as especialidades participaram da pesquisa, sendo sete de ginecologia e obstetrícia e seis de medicina de família e comunidade. O critério de inclusão era ser médico ou médica das residências em medicina de família e comunidade ou ginecologia e obstetrícia nas duas universidades públicas participantes do estudo. A fim de obter uma amostra não probabilística, utilizou-se a técnica de recrutamento em cadeia ou "bola de neve", na qual os(as) participantes do estudo indicam outros(as) participantes até que se atinja o ponto de saturação.

Resultados: Apesar de diferenças identificadas entre os(as) participantes, segundo os programas de residência, em relação à abordagem de gênero na formação médica e às suas repercussões na prática clínica, com maior apropriação pelos residentes de medicina de família e comunidade, sobressaem lacunas importantes na formação e no âmbito da graduação e especialização.

Conclusão: O conhecimento e o desenvolvimento de habilidades e técnicas baseadas em abordagem de gênero na formação médica são fundamentais para o exercício do cuidado integral que considera as conformações socioculturais dos(as) pacientes e suas implicações para o
\end{abstract} processo saúde-doença.

Palavra-chave: Gênero e Saúde; Identidade de Gênero; Educação Médica; Internato e Residência; Pesquisa Qualitativa.

\section{ABSTRACT}

Introduction: Medicine as an area of knowledge and practice has rendered invisible the importance of gender as an analytical category in medical education, as well as the impacts of gender differences and inequalities expressed in the context of clinical practice. Gender is recognized as a crucial aspect in medical education, mainly in the sense of promoting the quality of health care, considering gender differences in symptoms, risk factors for the disease and the care plan established in the context of the therapeutic relationship.

Objective: Based on this assumption, qualitative design research was conducted on the perception of training received on gender in the context of undergraduate and medical specialization of residents in Gynecology and Obstetrics and Family and Community Medicine at two public schools in the city of São Paulo.

Method: The research used the in-depth interview technique. In 2016, 13 residents of both specialities participated in the survey: seven from Gynecology and Obstetrics and six from Family and Community Medicine. The inclusion criterion was to be a doctor in a medical residency in Family and Community Medicine and Gynecology and Obstetrics at the two public universities participating in the study. The participants were accessed by the snowball recruitment technique, seeking a non-probabilistic sample, in which the study participants indicated other participants to the point of saturation.

Results: Despite the differences identified among the participants, according to the residency programs, concerning the gender approach in medical training and its repercussions in clinical practice, with higher appropriation by residents of Family and Community Medicine, essential gaps in training stand out, within the scope of undergraduate training and specialization.

Conclusion: Knowledge and the development of skills and techniques based on a gender approach in medical education are fundamental for the exercise of comprehensive care that considers the sociocultural conformations of patients and their implications for the health of the disease process.

Keywords: Gender and Health; Gender Identity; Undergraduate Medical Education; Graduate Medical Education.

1 Faculdade de Medicina, Universidade de São Paulo, São Paulo, São Paulo, Brasil.

${ }^{2}$ Associação Saúde da Família, São Paulo, São Paulo, Brasil.

Editora-chefe: Daniela Chiesa $\quad$ Editora associada: Daniela Chiesa

Recebido em 29/07/20; Aceito em 04/01/21. | Avaliado pelo processo de double blind review. 


\section{INTRODUÇÃO}

Apesar do crescente corpo de evidências sobre a importância da relação gênero e saúde em termos de padrões de morbimortalidade e manejo clínico ${ }^{1}$, essa relevância ainda é pouco considerada nos campos da produção do conhecimento e da formação dos(as) profissionais de saúde. Na direção do reconhecimento de tal importância, assiste-se, nas últimas duas décadas, ao crescente debate entre as humanidades e a saúde em torno de questões sobre como estereótipos e normas de gênero interferem nas práticas assistenciais de saúde voltadas ao cuidado de indivíduos e populações ${ }^{2}$. No Brasil, na última década, estudos acerca das questões de gênero e sexualidade no desenvolvimento de competências relacionadas ao cuidado integral em saúde e promoção dos direitos humanos ganham destaque, seja em pesquisas que analisam os currículos médicos ${ }^{3,4}$, em análises sobre a formação médica para o cuidado destinado à saúde da população LGBT, na perspectiva de médicos(as) que atuam na atenção básica ${ }^{5}$ ou nas experiências de estudantes de Medicina em cursos de extensão nas temáticas de sexualidade e gênero ${ }^{6}$.

SegundoVerdonketal. ${ }^{7}$, Bleakley ${ }^{8}$ eHammarström et al. ${ }^{9}$, as questões de gênero na saúde e na doença precisam ser abordadas na formação dos(as) futuros(as) médicos(as), considerando-se ao menos três grandes aspectos críticos. Em primeiro lugar, as pesquisas clínicas têm sido acusadas de serem "cegas a gênero" (gender-blind), por não terem a referência sobre orientação sexual, ou seja, as diferentes formas de atração afetiva e sexual de cada um(a) dos(as) participantes sob consideração, mesmo quando pertinentes. Em segundo lugar e estreitamente relacionado ao primeiro, o corpo de conhecimento produzido pela biomedicina sobre saúde e doença ainda é predominantemente produzido a partir dos homens, tomados como padrão universal humano. Nesse quesito, a literatura discute como, em estudos clínicos, participantes do sexo feminino foram historicamente excluídos por razões como: os efeitos de confusão do ciclo menstrual sobre os resultados dos testes, aumentando assim a complexidade e os custos de pesquisa; o receio de que o tratamento investigado afete a fertilidade feminina ou a gravidez; e a maior incidência de algumas doenças entre homens. O terceiro ponto crítico diz respeito ao papel do gênero e à sua influência na maneira como a cultura médica lidou (e permanece lidando) com sua abordagem nos contextos clínicos, de forma a reproduzir estereótipos de gênero para homens e mulheres com importantes impactos para a adesão aos tratamentos e o sucesso deles.

A formação em medicina, entendida como uma prática cultural, opera em duas frentes: a ciência médica, como campo de produção do conhecimento sob as bases da biomedicina, e a educação médica, que se orienta para as finalidades sociais da profissionalização do "ser" médico ${ }^{10}$. Considerando-se as conexões entre essas frentes, as escolas médicas necessitam de uma visão mais clara sobre a relevância específica das dimensões de gênero na formação de seus/suas alunos(as) ${ }^{7,8}$. Assim, seja na abordagem clínica das doenças em diferentes especialidades, como na farmacologia, em temas médicosociais como violência doméstica e sexual, em áreas como saúde sexual e reprodutiva, em domínios como a saúde mental (especialmente em tópicos como depressão, transtorno de estresse pós-traumático, transtornos de ansiedade e abuso de substâncias) ou em assuntos como a comunicação na relação profissional de saúde-paciente, a complexidade da expressão de gênero nas dimensões práticas e simbólicas da atuação em saúde merece atenção destacada.

O debate acerca da necessidade de problematizar diferentes aspectos relacionados a gênero na formação em Medicina tem como pano de fundo a ideia de que o currículo é uma experiência vivida; estende-se para além de programas ou listas de conteúdos teóricos e práticos. Como experiência vivida, o currículo constrói identidades ${ }^{11}$. Para Bleakley ${ }^{8}$, em educação médica, sendo corrente a imposição de metas de transição da identidade do estudante à do(a) médico(a), o processo de formação que não incorpora gênero reproduz sinais clássicos dos referentes masculinos na prática clínica, apoiados na defesa contra a admissão de incertezas e ambiguidades. Para Leite e Oliveira $^{10}$, o currículo em Medicina, como prática cultural, deve ser considerado como um híbrido cultural em constante movimento de recontextualização de textos, discursos e práticas ambivalentes, que não se esgotam nos discursos médicos e a eles não podem ser reduzidos; assim, a medicina, como prática profissional, é técnica-moral dependente ${ }^{12}$.

Segundo Bleakley ${ }^{8}$, o currículo formal cumpre papel central na modulação de concepções de gênero dos(as) profissionais de saúde ao longo de seu processo formativo, além de se constituir como uma experiência viva que serve à construção de identidades que pode (re)produzir vieses de gênero. O currículo oculto, por sua vez, inclui valores, crenças, normas e comportamentos presentes no ambiente educacional, mas não manifestos em documentos ${ }^{13}$. Vê-se, portanto, a importância de visibilizar e ampliar o debate sobre a importância da abordagem de gênero na formação médica, no que concerne aos aspectos relativos a gênero no exercício da profissão, como as diferenças nas oportunidades e a segregação dentro de especialidades ${ }^{14}$, o desenvolvimento de habilidades comunicacionais com usuários/pacientes ${ }^{15}$, o papel dos(as) professores(as) em defender a importância do debate sobre gênero ${ }^{16}$, entre outros. Uma vez que a equidade de gênero não é um processo espontâneo ${ }^{11}$, a mudança social que visa alcançar esse propósito precisa de investimento não apenas para os sujeitos na posição de pacientes/usuários da saúde, 
mas igualmente para profissionais que atuam em pesquisa e na prática clínico-assistencial, na formulação e gestão de serviços e em programas e atividades assistenciais.

Considerando a medicina como área de saber e prática que tem invisibilizado a importância de gênero como categoria teórica para a formação, bem como os impactos das diferenças e desigualdades de gênero no contexto clínico-assistencial, o presente trabalho objetiva analisar as percepções sobre a incorporação de gênero na formação teórico-prática vivenciada por residentes de medicina de família e comunidade (MFC) e de ginecologia e obstetrícia (GO) de duas universidades de São Paulo.

\section{MÉTODOS}

O presente artigo resulta das análises dos dados de pesquisa intitulada "Gênero na formação e na prática clínicoassistencial médica: estudo entre residentes de Medicina de Família e Comunidade e Ginecologia e Obstetrícia de duas universidades paulistas". O estudo foi realizado após a aprovação do Comitê de Ética em Pesquisa da instituição proponente (CAAE 62947616.8.0000.0065).

A pesquisa seguiu a metodologia qualitativa aplicada à saúde. A produção dos dados empíricos se norteou pela técnica de entrevista em profundidade, dada sua potencialidade de captar, a partir de um roteiro predefinido flexível e da interação pesquisador(a)-entrevistado(a), narrativas dos(as) participantes com o objetivo de alcançar a dimensão subjetiva de suas práticas e as concepções e os valores que as sustentam nos planos pessoal e profissional ${ }^{17,18}$. O roteiro das entrevistas foi organizado em blocos de perguntas que visavam: 1. entender como a perspectiva de gênero foi ou (não) oferecida e vivenciada na graduação e na residência de modo teórico e prático, e 2. verificar a sua pertinência e contribuição no âmbito de suas atuais vivências profissionais relacionadas à temática de gênero. Antes do início da pesquisa empírica, foram realizadas duas entrevistas-piloto com residentes de outras especialidades médicas que serviram ao propósito de ajuste do roteiro.

Como critérios de inclusão, consideraram-se homens e mulheres cursando residência médica em MFC ou GO, que aceitassem participar do protocolo de investigação. As entrevistas foram realizadas entre abril e agosto de 2016, após leitura, esclarecimento e assinatura do Termo de Consentimento Livre e Esclarecido (TCLE) pelos(as) participantes. A fim de obter uma amostra não probabilística, utilizou-se a técnica de recrutamento em cadeia ou "bola de neve", na qual os(as) participantes do estudo indicam outros(as) participantes até que se atinja o ponto de saturação. A escolha dessa estratégia se deveu ao seu custo-benefício e à sua praticidade, além de estar alicerçada em pressupostos da abordagem qualitativa que se ancoram na diversidade e não na representatividade amostral ${ }^{19}$. Os(as) primeiros(as) participantes foram acessados no âmbito dos dois cursos de residência de universidades públicas de São Paulo, por indicação dos(as) respectivos(as) coordenadores(as) dos programas de residência. Na sequência, cada entrevistado(a) indicava mais um ou dois residentes que eram convidados a participar da pesquisa.

Realizaram-se 13 entrevistas, sendo setecom profissionais que cursavam especialização em GO e seis em MFC. Todas as entrevistas foram conduzidas por duas discentes do curso médico que receberam treinamento em pesquisa qualitativa e na aplicação da técnica de entrevista em profundidade. As entrevistas foram realizadas individualmente, em local definido pelos(as) entrevistados(as), com média de duração de 50 minutos, e gravadas a partir da permissão dos(as) participantes. Após a transcrição dos áudios na íntegra, verificou-se a acurácia dos registros. O número final de entrevistados(as) foi definido no processo contínuo do trabalho de campo, por meio do critério de saturação. Na sequência, o processo analíticointerpretativo do material empírico foi realizado por meio da análise de conteúdo temático ${ }^{20}$.

$\mathrm{Na}$ análise, focalizaram-se temas previstos no roteiro e temas emergentes, e, para ambos, adotaram-se quatro etapas no processo de análise: 1. escuta repetida de cada entrevista processo de impregnação dos relatos; 2 . delimitação dos temas prioritários para análise; 3. síntese preliminar dos principais achados em cada um dos temas delimitados; e 4. confrontação dos achados empíricos primários com a literatura relativa aos temas da pesquisa e à fundamentação teórica gênero e formação médica. Desse processo, emergiram duas categorias de análise: 1. percepção e conhecimento do conceito e da abordagem de gênero, e 2. manejo teórico-prático do conceito e da abordagem de gênero na graduação e residência médicas. Na sequência, apresentaremos os resultados e a discussão das categorias de análise. Serão destacadas diferenciações entre participantes segundo a formação em MFC e GO. Salientamos que a nossa amostra não permitiu análises que contemplassem os aspectos de gênero e orientação sexual dos(as) participantes e suas particularidades quanto às percepções sobre os temas propostos na investigação.

\section{RESULTADOS E DISCUSSÃO}

O Quadro 1 apresenta a caracterização dos(as) entrevistados(as). Os nomes foram substituídos pela letra " $E$ " (entrevistado) acompanhada de um número, com o intuito de preservar a identidade dos(as) discentes. Os(as) participantes se formaram em oito faculdades médicas, o que demonstra a diversidade entre os(as) entrevistados(as) quanto às instituições de formação. Conforme o ano de formação de cada um(a) deles(as) expressa, são todos(as) médicos(as) jovens que ainda 
Quadro 1. Caracterização dos(as) participantes

\begin{tabular}{cccccc}
\hline Participantes & Gênero & Faculdade de graduação & Ano de formação & Faculdade de residência & Especialidade \\
\hline E1 & Feminino & Famerp & 2011 & UP1 & GO \\
\hline E2 & Feminino & FMUSP & 2014 & UP1 & GO \\
\hline E3 & Feminino & Unilus - Santos & 2013 & UP1 & GO \\
\hline E4 & Feminino & Unifesp & 2013 & UP2 & GO \\
\hline E5 & Masculino & Unifesp & 2014 & UP2 & GO \\
\hline E6 & Masculino & Unifesp & 2014 & UP2 & GO \\
\hline E7 & Feminino & Ufba & 2015 & UP1 & GO \\
\hline E8 & Feminino & UFPR & 2014 & UP1 & MFC \\
\hline E9 & Feminino & FMUSP & 2014 & UP1 & MFC \\
\hline E10 & Feminino & Santa Casa -SP & 2015 & UP1 & MFC \\
\hline E11 & Masculino & Uepa & 2015 & UP1 & MFC \\
\hline E12 & Feminino & PUC Campinas & 2015 & UP1 & MFC \\
\hline E13 & Masculino & PUC Sorocaba & 2015 & UP1 & MFC \\
\hline
\end{tabular}

vivenciam um processo de construção de seu eu-médico e com memórias recentes da graduação.

O conhecimento sobre gênero e a distinção sexo-gênero não foi homogêneo: enquanto alguns/algumas apontaram sexo como uma definição estabelecida desde a perspectiva biológica e gênero como uma identificação a partir de padrões socialmente definidos, outros(as) demonstraram confusão entre os dois conceitos. Apesar da pouca compreensão sobre a distinção entre sexo biológico (macho, intersexual, fêmea), identidade de gênero (cisgênero e transexual) e expressão de gênero (masculino, feminino e não binário), as duas últimas foram referidas pela totalidade dos(as) entrevistados(as) como uma construção que revela padrões socialmente estabelecidos.

Gênero eu acho que é uma construção que cria padrões e você acaba se identificando com esses padrões. Eque nada tem a ver com seu sexo biológico e que nada tem a ver com a expressão de afeto e sexualidade. $E$ identidade de gênero é isso, como a pessoa se enxerga nessas diferentes nuances (E13, masculino, MFC).

Chamou a atenção, em outro polo, que nenhum dos(as) participantes problematizou definições biológicas, como sexo, como parte da construção de um discurso científico também influenciado por padrões culturais, sociais e históricos ${ }^{21,22}$. Assim, médicos e médicas formados por diferentes escolas médicas, cursando residência em especialidades nas quais a relação gênero-saúde se interpõe de modo incisivo e cotidiano, pouco se conectam às discussões recentes que avançam de uma compreensão separada de sexo (biologia) e gênero (cultura) para outra que possibilita análises que conectam os processos biológicos e sociais ${ }^{23}$.

Outro aspecto em destaque foi a ausência de relatos espontâneos ou induzidos por perguntas presentes no roteiro de entrevista que situassem o masculino como construção histórica e social relacionada ao gênero. A totalidade das falas dos(as) participantes sobre gênero esteve voltada às mulheres e não aos homens, mesmo quando instados(as) a apresentar exemplos sobre os conteúdos sobre gênero no currículo formal e no oculto, bem como quando se solicitaram relatos de experiências de abordagem de gênero, consideradas exitosas ou não, no contexto clínico-assistencial. Apesar de uma abordagem essencialmente binária adotada nas narrativas dos(as) residentes (homem-mulher equivalente a masculinofeminino), nenhum/nenhuma mencionou ativamente a masculinidade como um aspecto constituinte do sistema de gênero. Essa ausência parece dizer respeito à lacuna temporal dos estudos que abordam os homens, em suas diversidades, como objeto pela saúde, desde a perspectiva construtivista e relacional de gênero; isso reforça, como apontado por Machin et al. ${ }^{24}$, que as masculinidades não são conceituadas como potenciais determinantes em saúde, em oposição ao feminino, que tem a abordagem em saúde como um espaço já estabelecido. De fato, a relação entre masculinidades e saúde, como agenda mais recente de pesquisa na saúde (que refuta a utilização da noção de homem genérico ou como uma categoria predominante nos estudos biomédicos) que busca os particularismos das formas de ser homem e a relação com os processos de saúde-adoecimento e cuidado ${ }^{25}$, permanece distante do universo dos(as) entrevistados(as).

Em relação à presença da abordagem de gênero na graduação de Medicina no Brasil, as Diretrizes Curriculares Nacionais (DCN), vigentes no período em que os(as) participantes cursaram a graduação, não explicitam a abordagem temática de 
gênero, mas trazem como perfil do egresso um(a) profissional com formação generalista, humanista, crítica e reflexiva, capacitado(a) a atuar pautado(a) em princípios éticos na promoção da saúde integral do ser humano ${ }^{26}$.

Como a identidade e as expressões de gênero são indissociáveis da singularidade de cada pessoa, havia a expectativa de que o tema fosse incorporado nos projetos pedagógicos dos cursos médicos, nos quais os(as) participantes se formaram. Contudo, foi consenso que suas faculdades de origem sistematicamente ignoravam a temática. Quando convidados(as) a lembrar-se de algum tipo de inserção da temática, poucos(as) referiram ocasional abordagem em aulas da psiquiatria, ainda assim centrada em patologias, ou em GO, com um foco em anatomia. Esse dado se coaduna com a percepção de que seus/suas professores(as) na graduação não tinham interesse ou não reconheciam a importância da temática.

Eu lembro de uma aula que tocou nesse assunto em gineco. Em psiquiatria eu não vi nada disso. $\mathrm{Na}$ verdade, em psiquiatria era muito mais colocar isso num CID do que conversar sobre isso... Na faculdade acho que gênero foi muito pouco abordado (E8, feminino, MFC).

Na faculdade, [a abordagem] não existiu. Foi mais na pesquisa que eu fiz. A gente precisa fazer uma monografia pra terminar a faculdade e a minha monografia foi sobre isso. Ninguém entendeu. Inclusive na minha apresentação da monografia os professores que estavam lá para me avaliar não entenderam (E7, feminino, GO).

Mesmo considerando as atividades extracurriculares que fazem parte do chamado currículo paralelo, como ligas acadêmicas e projetos de extensão, foram poucos os relatos de abordagem da temática de gênero nestes espaços. Diante da ausência de formação conceitual-teórica em gênero em atividades curriculares e extracurriculares, alguns/algumas entrevistados(as) construíram narrativas que colocam na vivência pessoal a possibilidade de algum conhecimento da temática gênero:

Eu tenho a impressão que os chefes eles acham que tudo isso, quanto a qualquer coisa, está subentendido em como ser médico. Como se isso tivesse sido abordado bem na graduação, só que não foi. Aí o que acontece, cada um se vira baseado na sua própria história, e no que acha que deve fazer (E4, feminino, GO).

Por causa da ausência da abordagem de gênero na graduação, os(as) entrevistados(as) se dividiram em relação à sua importância como conteúdo obrigatório no currículo médico. Para os(as) residentes em GO, seria o contato mais frequente com o tema da sexualidade que os(as) levaria a tratar desse assunto com mais "naturalidade" e sem necessidade de embasamento teórico-conceitual em questões e temas relacionados a gênero. Já os(as) residentes de MFC consideraram gênero como um marcador social do adoecimento, trazendo espontaneamente em seus relatos a referência de que a prática clínica é essencialmente "generificada":

A gente não é médico que vai lá e lida com a doença da pessoa, a gente lida com tudo. E todas as questões de gênero são questões que geram muitas doenças, porque a violência de gênero, a discriminação... tudo isso pode levar a doenças psíquicas e até biológicas. Então assim, o viés de gênero é superimportante e a gente tem que aprender a lidar (E12, feminino, MFC).

Também foram os(as) residentes de MFC quem mais mencionaram os cenários extracurriculares como espaços propícios (ou únicos) para discussão de gênero durante a formação, já que, na estrutura das disciplinas formais, a ausência do tema está dada. Os espaços extracurriculares por eles/elas nomeados foram aqueles de auto-organização de mulheres, como coletivos feministas, ou de grupos políticos socialmente minoritários, como coletivos LGBTs. Ademais, os(as) residentes de MFC chegaram a apontar que não haverá uniformização da conceituação de gênero sem que haja uma política institucional que trabalhe a incorporação da temática nas escolas médicas, sendo o exemplo maior usado para tal argumento a capacidade empática por parte das médicas mulheres diante de situações impostas por violência de gênero, em contraponto com à postura adotada por médicos homens.

A reflexão sobre como e quanto a falta de espaços institucionais de discussão sobre a temática de gênero acarreta o exercício da relação médico-paciente e da tomada de decisão na prática clínica ancorado e dependente exclusivamente da vivência pessoal sobressaiu entre residentes de MFC. Alguns/ algumas chegaram a pontuar queo(a) usuário(a) ficaria vulnerável ao grau da capacidade pessoal-subjetiva do(a) médico(a) em abordar suas demandas com delicadeza e eficácia.

Eu acho que é essencial. Eu acho que precisa estar na faculdade de medicina, precisa ter tempo pra refletir sobre isso, precisa ter gente boa, gente que faz discussão sobre o tema para não ficar uma discussão rasa [...] até porque o médico cumpre o papel de formador de opinião (E10, feminino, MFC).

No contexto da pós-graduação lato sensu, faz-se necessário pontuar que os pré-requisitos definidos pela Comissão Nacional de Residência Médica (CNRM) no país para os dois programas analisados também não mencionam explicitamente a perspectiva de gênero ${ }^{27}$.

No cenário da residência, a abordagem de gênero (podendo ser composta por aulas, discussões ou textos de referências adotados) também se apresentou de modo distinto 
para os(as) residentes de MFC e GO. Entre os(as) de MFC, a abordagem de gênero no contexto da atenção primária à saúde (APS) é mencionada em cenários concretos de prática clínica, como discussões de casos clínicos, bem como em aulas teóricas e em termos programáticos da estruturação e regulação da MFC como especialidade:

Ofato de a Sociedade Brasileira de Medicina de Família [e Comunidade] ter um grupo de trabalho, ter um GT formal, que se presta a discutir sexualidade, questão de gênero [...] isso já é um avanço. São de médicos de família as principais políticas mais inclusivas nesse sentido (E13, masculino, MFC).

Por sua vez, os(as) residentes em GO relataram não haver abordagem formal de gênero, e suas percepções enfatizam unicamente a experiência prática como mecanismo de aprendizado. Somado a isso, quando questionados(as) sobre como percebem a reação de colegas da residência quanto à temática, muitos(as) alegaram perceber desinteresse ou mesmo desgosto: "Na verdade a maior parte das pessoas têm receio de abordar esse assunto, porque não sabem lidar com isso. As pessoas, às vezes, não querem nem ouvir, preferem deixar de lado e já ouvi professor falando que é safadeza" (E5, masculino, GO).

A percepção da aplicabilidade da abordagem de gênero na prática clínica foi unânime entre os(as) residentes de MFC, destacando-se tanto no sentido de gênero ser um dos marcadores do processo saúde-doença quanto no sentido de a abordagem ser necessária para o estabelecimento do vínculo entre médico e paciente e para a determinação de condutas e da prática médica. Entre os(as) residentes de GO, parte apontou a necessidade de conhecimento do tema para uma prática clínica adequada, considerando que o país vive uma conjuntura social e política em que temáticas como gênero e sexualidade são cada vez mais presentes no cotidiano da saúde.

Se você estiver muito resistente, você vai estar sendo no mínimo danoso ao seu paciente. Fora o estresse que você vai gerar para eles, por não se sentir acolhido, você vai carregar consigo um embate muito forte contra as suas ideias, ninguém consegue levar esse embate muito a fundo, você não consegue deixar de atender um transgênero, deixar de atender uma questão sexual de uma menina de 10 anos que já começou a vida sexual. Não cabe a nós julgar, cabe a nós orientar (E6, masculino, GO).

Apesar do reconhecimento da aplicabilidade da temática na assistência médica por alguns/algumas, outros(as) residentes em GO questionaram a real necessidade da abordagem em gênero para o cuidado médico.

Sendo bem cru, sendo bem cientificista, inicialmente a gente não deve, ou não é tão importante assim, a gente identificar o gênero de alguém. Num contexto médico mais fisiologista, mais patológico da coisa, mais orgânico da coisa, eu acredito que não faz tanta diferença assim. A menos que, claro, tem que ser levado caso a caso, tem que ser discutido se isso faz alguma diferenciação na clínica (E6, masculino, GO).

Como se pode apreender dos últimos relatos, após a graduação, a abordagem de gênero assumiu rumos diferentes de acordo com a especialidade médica seguida. Os(as) residentes de MFC apontaram que as discussões sobre gênero, e particularmente sobre a autonomia da mulher, são constantes ao longo da especialização, presentes em aulas e discussões de casos, o que os(as) torna preparados(as) para lidar com essas questões no contexto assistencial. Os(os) residentes em GO, ao contário, consideraram que a base para a abordagem de gênero se dá na prática, uma vez que estão em contato direto com o feminino. A postura dos(as) entrevistados(as) de GO se aproxima da postura dos(as) alunos(as) norte-americanos(as), como evidenciado por Henrich et al. ${ }^{28}$ em survey com estudantes de Medicina. Nessa pesquisa, os(as) estudantes avaliaram a presença de gênero como componente curricular como fraca a moderada, enquanto a percepção de sua capacidade de atuação no atendimento às mulheres foi superiormente avaliada, de maneira semelhante à relatada pelos entrevistados nesta pesquisa. Delegar à prática clínica a capacitação para a abordagem de gênero não resulta, como apontado por Henrich et al. $^{28}$, na capacidade de estabelecimento de vínculo ou empatia com os(as) pacientes.

Nesse sentido, é necessário apontar a diferenciação entre viés de gênero e percepção de gênero na educação médica, já estabelecida por Verdonk et al.7. Enquanto o viés de gênero é atribuído apenas às especificidades de gênero aplicáveis num contexto clínico-biológico, a percepção de gênero abarca o contexto sociocultural que determina as relações sociais entre sujeitos. Sendo assim, há a necessidade de que o viés de gênero na formação médica inclua também a percepção de gênero. Tendo isso em mente, é possível afirmar que um viés de gênero atrelado exclusivamente ao contexto biológico é contemplado nas escolas médicas em que os(as) participantes desta pesquisa se formaram. Tal afirmação pode ser estendida para a residência em GO, enquanto a residência em MFC ultrapassa as expectativas relacionadas ao ensino médico, por ter abraçado uma perspectiva de gênero ampliada para estudo do processo de adoecimento e para direcionamentos de condutas clínicas mais integrais.

A discrepância entre a abordagem de gênero nas residências médicas tem origens múltiplas, abarcando desde interesses individuais até programática da especialização, 
mostrando-se mais presente no contexto da MFC comparativamente à GO. Isso possivelmente está atrelado ao fato de a MFC ser uma especialidade generalista que trabalha com o método clínico centrado na pessoa ${ }^{25}$, de modo que a abordagem de gênero deve necessariamente englobar as conformações socioculturais do(a) paciente e suas implicações no processo saúde-doença. Como resultado, há maior valorização do gênero como aspecto aplicável na prática clínica - tanto enfocando relação médico-paciente quanto condutas - dessa especialidade. Ao contrário, na GO observouse abordagem mais biologicista, apesar do reconhecimento de gênero como marcador social do adoecimento.

\section{CONSIDERAÇÕES FINAIS}

As pesquisas de gênero em saúde constituem um complexo e relacional campo com grandes possibilidades de desenvolvimento. Complexo, pois engloba temas da saúde, do adoecimento e do cuidado que tanto podem ser abordados, de forma independente, no interior dos campos da saúde e das ciências humanas e sociais como basear-se em uma perspectiva de aproximação e colaboração entre eles. É relacional, e com grandes possibilidades de desenvolvimento, na medida em que diferentes questões têm sido discutidas, perpassando os processos de adoecimento e morte, os modos de assistência e cuidado em saúde, as formulações de políticas sociais voltadas a populações de homens e mulheres e, ainda, a formação de profissionais para atuação em saúde.

Tal como observado neste estudo, a ausência da temática de gênero na graduação em Medicina mostra-se sistemática nos cursos médicos em escalas nacional ${ }^{3-6}$ e internacional ${ }^{7,8,13}$. Bleakley ${ }^{8}$ aponta que a discussão de gênero na educação médica está atrelada de forma restritiva a análises demográficas e sociais, questões essas abordadas na carreira médica de forma biologizante. Da mesma forma, Henrich et al. ${ }^{28}$ demonstraram que, por meio de uma pesquisa em 101 escolas médicas dos Estados Unidos, os(as) alunos(as) identificaram que as únicas abordagens sobre gênero eram delegadas a disciplinas como GO ou relegadas a disciplinas pouco tradicionais e não participantes do currículo formal. Isso demonstra grande similaridade entre os dados coletados empiricamente nesta pesquisa com aqueles produzidos em universidades norteamericanas, evidenciando uma padronização da forma como o gênero é trabalhado na educação médica, que pode ser devido à relação retórica entre a educação médica brasileira e os modelos norte-americanos.

Os achados da pesquisa demonstram que 0 conhecimento acumulado durante a graduação não é suficiente para que as questões de gênero sejam abordadas de forma a influenciar planos terapêuticos no contexto da residência médica, podendo esse déficit ser maléfico para a prática clínica e para o estabelecimento de vínculo na relação médico-paciente ${ }^{29}$. Além disso, foi levantado questionamento sobre o papel social que o(a) médico(a) cumpre, apontando que faz parte desse papel formar opinião sobre o assunto, de modo que a perspectiva de gênero na formação cumpriria funções para além da prática clínica per se.

A análise das entrevistas permitiu identificar aspectos quanto à abordagem de gênero na formação médica e às suas repercussões na prática clínica em especialidades essencialmente distintas, a MFC e GO. Essas diferenças, que tangenciam as características técnicas específicas de cada programa de residência, não impactaram a conceituação de gênero e as temáticas prioritariamente referidas às questões de gênero entre os entrevistados. No entanto, demonstram ser impactantes no que concerne ao entendimento da importância do viés e da percepção de gênero na formação médica, na prática clínica e na construção da relação médico-paciente, vindo a influenciar o sentido e a aplicabilidade de conteúdo de gênero no contexto clínico-assistencial pelos(as) médicos(as) entrevistados(as).

As diferenças na abordagem de gênero nas especialidades de MFC e GO não repercutiram na capacidade de conceituação dos(as) residentes entrevistados, que adotaram uma abordagem essencialmente binária e focada na generificação do feminino, fortemente atrelada às questões de sexualidade ou violência de gênero. Evidenciou-se, a partir das falas dos(as) paticipantes, que os currículos médicos sistematicamente ignoram gênero como aspecto relevante para a formação, ao relegarem a temática a disciplinas com enfoque restritos à reprodução. Essa abordagem mostra que o viés de gênero nas escolas médicas é perpassado por um olhar estereotipado e biologista ${ }^{3}$, em consonância com o observado em escolas médicas internacionais ${ }^{16,28}$.

A discrepância entre as abordagens de gênero nas residências médicas tem origens múltiplas, abarcando desde interesses individuais até programática da especialização, mostrando-se mais presente no contexto da MFC comparativamente à GO. Isso possivelmente está atrelado ao fato de a MFC ser uma especialidade generalista que trabalha com o método clínico centrado na pessoa ${ }^{30}$, de modo que a abordagem de gênero deve necessariamente englobar as conformações socioculturais do(a) paciente e suas implicações no processo saúde-doença. Como resultado, há maior valorização do gênero como aspecto aplicável na prática clínica - enfocando tanto a relação médico-paciente quanto as condutas - dessa especialidade do que em GO, que adota uma abordagem mais biologicista, apesar do reconhecimento de gênero como marcador social do adoecimento.

O presente estudo também evidencia que gênero é 
indiscutivelmente reconhecido como um marcador social de diferença que impacta a produção de vulnerabilidades ${ }^{29}$ e a experiência do adoecimento, e que o desconhecimento da temática pode resultar em processos iatrogênicos por parte dos(as) médicos(as), justificando sua inserção como temática fundamental do currículo formal ${ }^{31,32}$. É necessário que os(as) estudantes tenham contato com o assunto durante a formação médica na graduação e nas áreas de especialização, sem que o assunto fique restrito a atividades relacionadas ao currículo oculto, para que haja uma efetiva e adequada capacitação para o cuidado integral destinado aos homens e às mulheres nos vários contextos clínico-assistenciais ${ }^{32}$. Além disso, diante de um quadro de reconhecimento de tamanhos e complexos diferenciais nos padrões de adoecimento, morte e cuidado quando se comparam os homens e as mulheres, a incorporação da abordagem de gênero na formação em Medicina se impõe, seja em sua dimensão da prática profissional (assistência) ou na produção do conhecimento (pesquisa).

\section{CONTRIBUIÇÃO DOS(AS) AUTORES(AS)}

Marcia Thereza Couto idealizou e coordenou a pesquisa empírica, delimitou o escopo do artigo e colaborou na redação de todas as suas versões do manuscrito. Rosana Machin idealizou e coordenou a pesquisa empírica, colaborou na delimitação do escopo do artigo e na redação da versão inicial do manuscrito. Maria Renata Mencacci Costa participou da idealização da pesquisa, conduziu a pesquisa empírica, analisou os dados produzidos e colaborou na delimitação do artigo e na redação de sua primeira versão. Ana Luisa Smith Rocha participou da idealização da pesquisa, conduziu a pesquisa empírica, analisou os dados produzidos e colaborou na redação de sua primeira versão. Ana Claudia Camargo Germani colaborou na redação da versão final do artigo. Antônio Augusto Modesto colaborou na redação da versão final do artigo.

\section{CONFLITO DE INTERESSES}

Os(as) autores(as) declaram não haver conflito de interesses neste estudo.

\section{FINANCIAMENTO}

O estudo contou com o apoio financeiro do Conselho Nacional de Desenvolvimento Científico e Tecnológico (CNPq) nas modalidades de bolsa de iniciação científica (Programa Institucional de Bolsas de Iniciação Científica Pibic) e bolsa de iniciação em desenvolvimento tecnológico e inovação (Programa Institucional de Bolsas de Inciação em Desenvolvimento Tecnológico e Inovação - Pibiti), no período de agosto de 2016 a janeiro de 2017, para Ana Luisa Smith Rocha (Pibic) e Maria Renata Mencacci Costa (Pibiti).

\section{REFERÊNCIAS}

1. Doyal L. Sex, gender, and health: the need for a new approach. BMJ. 2001 Nov 3;323(7320):1061-3.

2. Hankivsky O. Women's health, men's health, and gender and health: implications of intersectionality. Soc Sci Med. 2012 June 1;74(11):1712-20.

3. Raimondi GA, Moreira C, Barros NF. Gêneros e sexualidades na educação médica: entre o currículo oculto e a integralidade do cuidado. Saúde Soc. 7 out 2019.;28:198-209.

4. Raimondi GA, Abreu YR, Borges IM, Silva GB, Hattori WT, Paulino DB. Gênero e sexualidade nas escolas médicas federais do Brasil: uma análise de projetos pedagógicos curriculares. Rev Bras Educ Med. 2020;44(2) e046.

5. Negreiros FR, Ferreira BD, Freitas DD, Pedrosa Jl, Nascimento EF. Saúde de lésbicas, gays, bissexuais, travestis e transexuais: da formação médica à atuação profissional. Rev Bras Educ Med. mar. 2019;43(1):23-31.

6. Rufino AC, Madeiro AP, Girão MJ. O ensino da sexualidade nos cursos médicos: a percepção de estudantes do Piauí. Rev Bras Educ Med. jun 2013;37(2):178-85.

7. Verdonk P, Benschop YW, De Haes HC, Lagro-Janssen TL. From gender bias to gender awareness in medical education. Adv Health Sci Educ. 2009 Mar $10 ; 14(1): 135-52$.

8. Bleakley A. Gender matters in medical education. Med Educ. 2013;47:59-70.

9. Hammarström A, Johansson K, Annandale E, Ahlgren C, Aléx L, Christianson $M$, et al. Central gender theoretical concepts in health research: the state of the art. J Epidemiol Community Health. 2014 Feb 10;68(2):185-90.

10. Leite AF, Oliveira TR. Sobre educar médicas e médicos: marcas de gênero em um currículo de Medicina. Estud Fem. dez. 2015;23(3):779-801.

11. Sharma M. Applying feminist theory to medical education. The Lancet. 2019 Feb 9;393(10171):570-8.

12. Schraiber LB. No encontro da técnica com a ética: o exercício de julgar e decidir no cotidiano do trabalho em medicina. Interface Comun Saúde Educ. 1997;1:123-40.

13. Cheng LF, Yang HC. Learning about gender on campus: an analysis of the hidden curriculum for medical students. Med Educ. 2015 Mar;49(3):321-31.

14. Scheffer MC, Cassenote AJ. La feminización de la Medicina en Brasil. Rev Bioét. ago 2013;21(2):268-77.

15. Medeiros NS, Santos TR, Trindade EM, Almeida KJ. Avaliação do desenvolvimento de competências afetivas e empáticas do futuro médico. Rev Bras Educ Med. dez 2013;37(4):515-25.

16. Hamberg K. Gender bias in medicine. Women's Health (Lond). 2008 May;4(3):237-43.

17. Becker HS. Segredos e truques da pesquisa. Rio de Janeiro: Zahar; 2007.

18. DiCicco-Bloom B, Crabtree BF. The qualitative research interview. Med Educ. 2006 Apr;40(4):314-21.

19. Noy C. Sampling knowledge: the hermeneutics of snowball sampling in qualitative research. Int J Soc Res Methodol. 2008 Oct 10;11(4):327-44.

20. Fontana A, Frey JH. The interview: from structured questions to negotiated text. Handbook of Qualitative Research. 2000;2(6):645-72.

21. Laqueur T. Inventando o sexo. Rio de Janeiro: Relume Dumará; 2001.

22. Rohden F. A construção da diferença sexual na medicina. Cad Saude Publica. 2003;19:S201-12.

23. Hankivsky O, Doyal L, Einstein G, Kelly U, Shim J, Weber L, et al. The odd couple: using biomedical and intersectional approaches to address health inequities. Glob Health Action. 2017 Jan 10;10(Supp 2):73-81.

24. Machin R, Couto MT, Silva GS, Schraiber LB, Gomes R, Figueiredo WDS, et al. Concepções de gênero, masculinidade e cuidados em saúde: estudo com profissionais de saúde da atenção primária. Cien Saude Colet. 2011;16:4503-12.

25. Couto MT, Dantas SM. Gênero, masculinidades e saúde em revista: a produção da área na revista Saúde e Sociedade. Saúde Soc. 2016;25:857-68.

26. Brasil. Resolução $n^{\circ}$ 3, de 20 de junho de 2014. Institui Diretrizes Curriculares Nacionais do Curso de Graduação em Medicina e dá outras providências. Diário Oficial da União; 2014. Seção 1, p. 8-11. 
27. Brasil. Resolução no 2, de 17 de maio de 2006. Dispõe sobre requisitos mínimos dos Programas de Residência Médica e dá outras providências. Diário Oficial da União; 2006. Seção 1, p. 23-36.

28. Henrich JB, Viscoli CM, Abraham GD. Medical students' assessment of education and training in women's health and in sex and gender differences. J Women's Health. 2008 June 10;17(5):815-27.

29. Bourgois P, Holmes SM, Sue K, Quesada J. Structural vulnerability: operationalizing the concept to address health disparities in clinical care. Acad Med. 2017 Mar;92(3):299-307.
30. Stewart M, Brown JB, Weston WW, McWhinney IR, McWilliam CL, Freeman TR. Medicina centrada na pessoa: transformando o método clínico. Porto Alegre: Artmed; 2017.

31. Westerhaus M, Finnegan A, Haidar M, Kleinman A, Mukherjee J, Farmer P. The necessity of social medicine in medical education. Acad Med. 2015 May 10;90(5):565-8.

32. Springer KW, Stellman JM, Jordan-Young RM. Beyond a catalogue of differences: a theoretical frame and good practice guidelines for researching sex/gender in human health. Soc Sci Med. 2012 June 10;74(11):1817-24. 